\title{
Evaluation of Design Collaboration Tools for the SunStang Electric Car Project
}

\author{
Ralph O. Buchal, Dan Rogers, Pranay Krishen \\ Department of Mechanical \& Materials Engineering \\ The University of Western Ontario \\ London, Ontario, Canada \\ rbuchal@eng.uwo.ca
}

\begin{abstract}
Engineering design teams require a variety of collaboration tools to work effectively. The SunStang Electric Car student team at the University of Western Ontario has identified specific collaboration needs, and has surveyed and evaluated a variety of available tools including Yahoo! Groups, Microsoft Sharepoint, Google Apps, Microsoft Groove, Microsoft OneNote, and Solidworks PDMWorks. They have concluded that no single tool satisfies all of the requirements, and that a combination of tools is required. They have adopted Google Apps, Microsoft Groove and PDMWorks as their main tools.
\end{abstract}

\section{Introduction}

The SunStang Electric Car Project is a student project within the Faculty of Engineering at the University of Western Ontario. Since its inception as a Master's thesis in 1992, the project's mandate has been to design, construct and race solar powered electric vehicles at biennial international competitions. Most recently the team traveled to Australia, and raced 3020 $\mathrm{km}$ across the across the Outback, as part of the 2007 World Solar Challenge.

An important aspect of SunStang's operations has been the use of collaboration tools to effectively and efficiently manage design progression. This paper describes and evaluates the various tools SunStang has had experience with, and concludes with a summary of the tools the team has adopted for the 2009 design cycle.

\section{Information \& Data Types}

As an extracurricular undergraduate engineering project that must accommodate the varying schedules of students, SunStang's operations demand a simple to use, yet effective, infrastructure for inter-team communications and data sharing. At an organizational level, this infrastructure must facilitate schedule and workflow management such that team managers can issue and track tasks with minimal direct interaction between members. Furthermore, the system must offer an efficient portal for internal and external communication. Finally, the team should be able to share technical data and CAD models to support concurrent engineering.

The internal collaboration system should feature tools to communicate as a group, share files and documents, access databases in real time, and even share calendars and tasks. The external communication system should allow team members to share information with the outside world. In this case, the most widely accepted means of communication is via email, warranting the need for an integrated team email system.

\section{Team Collaboration Requirements}

The collaboration tools must be accessible via any internet connection, since many team members need to collaborate from off-campus or laptop connections. The tools must be user-friendly and well integrated. The tools should support the easy creation and management of user accounts by the team itself. All collaboration tools should be easy to use and accessible from a single software application or web portal. In addition to these general requirements, specific tools are required as described in the following sections.

3.1 Scheduling tools (calendar, tasks, reminders) Advanced scheduling tools should be available, allowing the users to maintain a personal calendar, as well as view a shared team calendar. A task list should 
be easily generated and viewed by all users; this list should generate reminders when deadlines are approaching.

\subsection{Discussion forums}

The tools should support both synchronous and asynchronous discussions. Live chat capabilities allow team members to communicate in real time. Threaded discussion boards allow members to post and read messages.

\subsection{Multimedia Document Creation}

Engineers communicate using a variety of text, mathematical notation, sketches, images and other media. Tools are needed to provide natural and seamless use of all of these forms of communication in a single multimedia document.

\subsection{Shared file repository}

A shared file repository is a key requirement. The shared repository must allow users to upload, share and manage team files of all types. Version control and check-in/check-out are desirable features. To be practical, the tool must not limit file size or total repository storage capacity.

\subsection{Shared document management}

The collaboration tool must support group document authoring. One method is sequential editing, with each member checking out the document, making revisions, and checking it back in before the next team member can work on it. An alternative method is live editing, with multiple people editing the same document simultaneously. SunStang finds both approaches useful in different circumstances. It is important to manage versions to ensure that everyone is working on the latest document.

\subsection{Product Data Management (PDM)}

$\mathrm{CAD}$ files are more difficult to manage than other forms of files due to the complex relationship between CAD files corresponding to the product structure. Important capabilities include: version control, access control, CAD integration, and viewing. Typically a Product Data Management (PDM) system is required to manage CAD files.

\subsection{Contact list}

The application must be capable of creating and organizing a master team list with all relevant contact information, as well as sub team lists for individual projects. Existing lists in various file formats such as .csv must be importable to the program and the program must be able to export lists in various formats.

\subsection{E-mail interface}

The program must interface with an email application such as gmail or Microsoft Outlook in order to simplify mass e-mails and e-mail archiving.

\subsection{User Identification and Change History Tracking}

Any shared tools should allow tracking of modifications and additions made by different team members. It should be easy to see who added or changed what, and when. In a live document editing session, all user actions should be clearly visible and traceable to specific members. For example, if someone adds a note or comment to a document, the identity of the comment author should be automatically indicated.

\section{Survey and Evaluation of Available Tools}

The SunStang team has evaluated several different collaboration tools to support their needs. This section describes the tools used, and provides a brief evaluation of each tool.

\subsection{Yahoo! Groups}

Yahoo! Groups [1] allows users to create free accounts, and provides access to forums that facilitate group discussions. Generally speaking, the forum style is better suited for larger teams with heavy discussion traffic. While it is possible to integrate free @yahoo.com email accounts, there is no option to utilize a private domain name (such as @sunstang.ca). It is, however, possible to request email notifications outlining changes to the discussion boards. Overall, Yahoo! Groups offers a basic collaboration tool that effectively facilitates internal team discussions with no upfront investment.

On the downside, Yahoo!'s GUI is somewhat rudimentary and features prominent advertising. Basic file sharing and calendar functions are available, but they do not meet the storage size and navigational flexibility required by SunStang. 


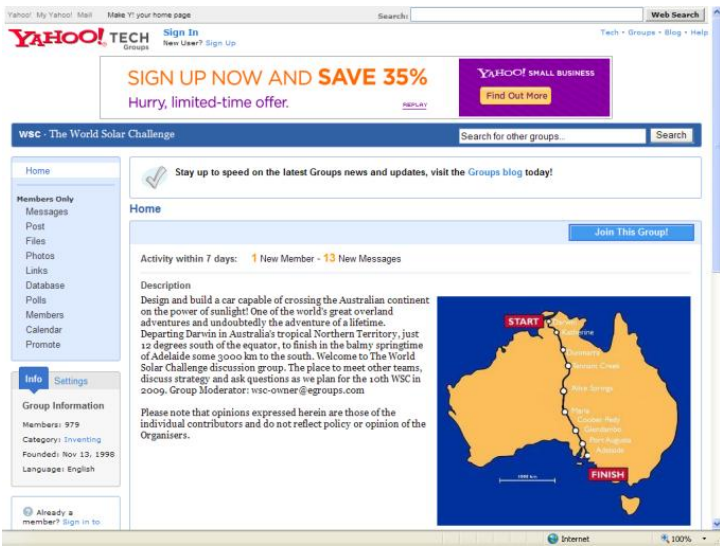

Figure 1. Example Yahoo Group site.

\subsection{Microsoft SharePoint}

SharePoint [2] is a server-based application for webbased collaboration and document management. Amongst its greatest strengths is its ability to interface with other Microsoft products. By deploying SharePoint within a Microsoft Active Directory infrastructure, it is convenient to manage user profiles and permission levels. Furthermore, direct integration with Microsoft Exchange Server provides a powerful and easy-to-use communication, scheduling, task management and contact-sharing portal. With the added ability to serve shared Microsoft OneNote documents, SharePoint fulfills the real-time document editing capability. Tools for team discussions, announcements, workflow management, and file sharing capabilities are built into the customizable SharePoint portal. Overall SharePoint offers a simple, clean GUI, as shown in Figure 2.

A significant disadvantage of SharePoint for student teams is its dependence on intensive (and flexible) IT support. SunStang has had limited access to a SharePoint server maintained by the Western Engineering IT Group, but the level of support has been insufficient to leverage some of the most important capabilities of SharePoint - especially the ability to manage Active Directory. The existing SharePoint server provides easy access through existing student engineering accounts, but adding accounts for members other than engineering students must be done by the Engineering IT staff.

Though SharePoint provides check-in/out versioning control, it currently does not support CAD file management. Therefore, it is necessary to supplement SharePoint with a third-party software such as PDMWorks.

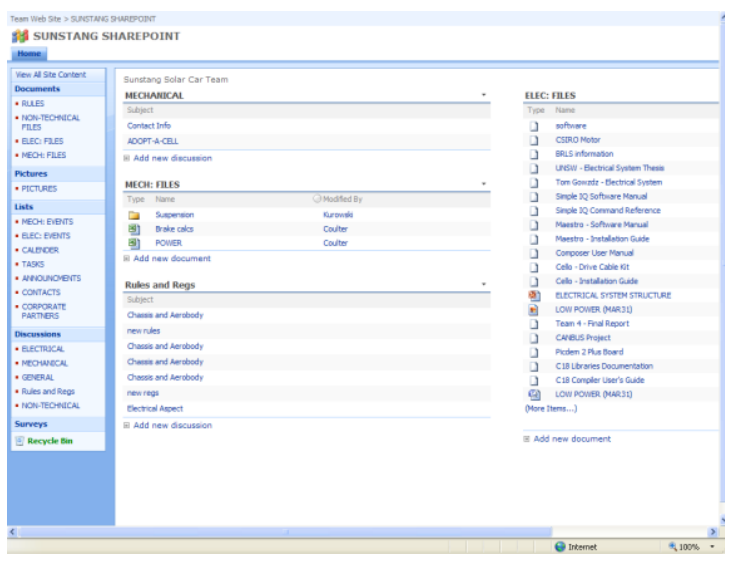

Figure 2. SunStang SharePoint site.

\subsection{Google Apps}

Google has been rapidly developing their communication and collaboration applications since Gmail was released in April of 2004. In the past year, Google has released its latest project, Google Apps [3], which allows organizations to link an existing domain name (sunstang.ca) to Google's own server. This very powerful combination allows an organization to setup and administer its entire email server directly through Google, while utilizing its own domain name. Email server upkeep is simplified through the Google Apps domain management interface and provides a $99.7 \%$ uptime.

In addition to the email feature, Google Apps provide a comprehensive set of collaboration tools that can be selected as "widgets" and published onto a customized start page. Team members can then set this start page as the default homepage (http://team.sunstang.ca/) of their browser, and have access to their SunStang email account, discussion forum, document database, calendar of events, and even an internal SunStang chat interface. Figure 3 shows a screenshot of the customizable Google start page that every user would see when signing in.

\footnotetext{
${ }^{1}$ A standardized on-screen representation of a control that may be manipulated by the user.
} 


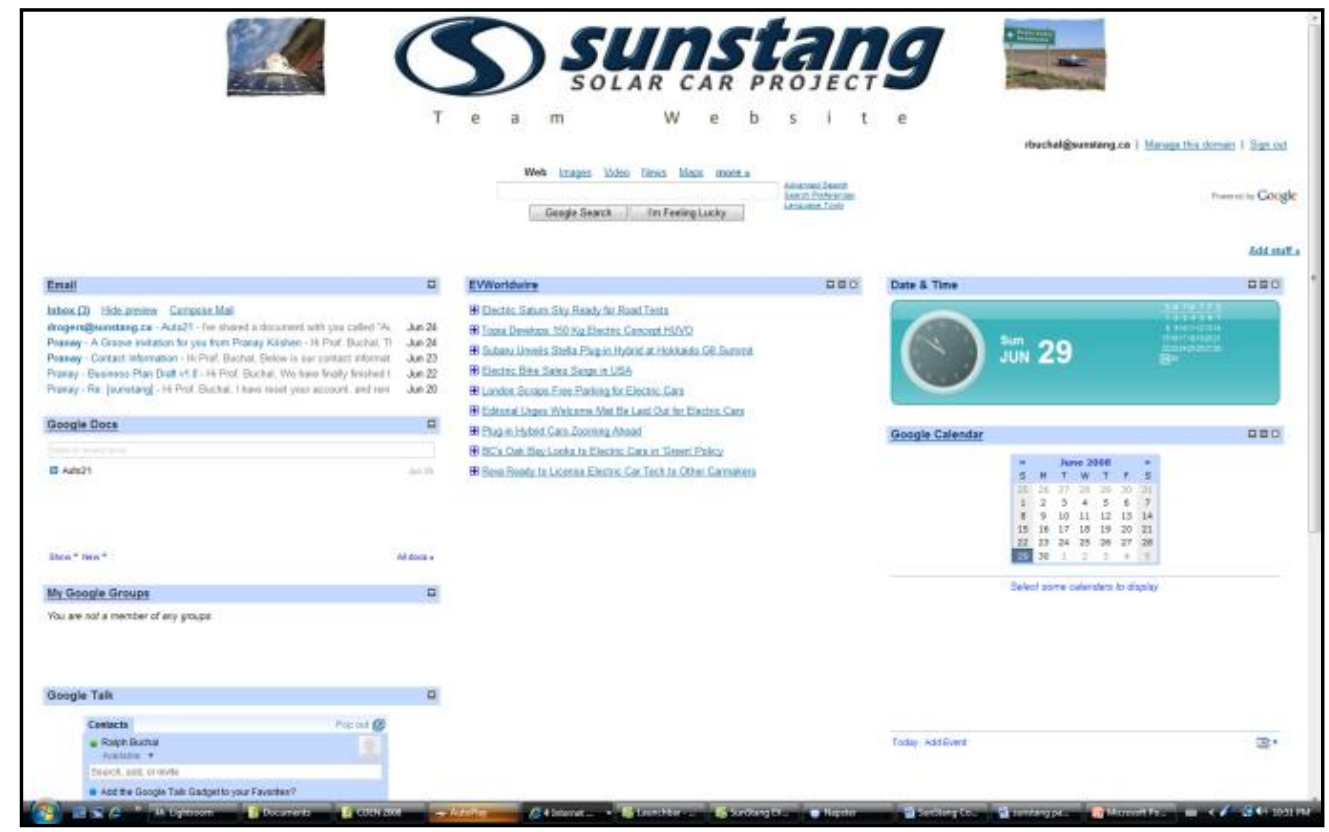

Figure 4. Sunstang team website on Google Apps (http://team.sunstang.ca).

The start page provides a collection of customizable and easy-to-use collaboration tools. Google Groups is the application which allows users to contribute to and view the forum, and upload files. The Email link allows the user to quickly access their @ sunstang.ca email account which is easily generated for them. Google Docs allows multiple users to work on a single live document. The calendar application allows users to create their own calendar, as well as view the master team calendar. The user can also add personal ${ }^{2}$ widget applications such as the clock shown in the figure.
A significant drawback of the Google option is the inability to upload files to an online storage facility. However, this functionality can be added by creating an FTP link (within the Bookmarks Widget) to SunStang's central server. Though this would require users to enter an additional username and password, it would provide access to all SunStang files.

\subsection{Microsoft Groove}

Microsoft Groove [4] is a Microsoft Office product designed primarily for peer-to-peer collaboration and file sharing. Groove is based on a Windows client

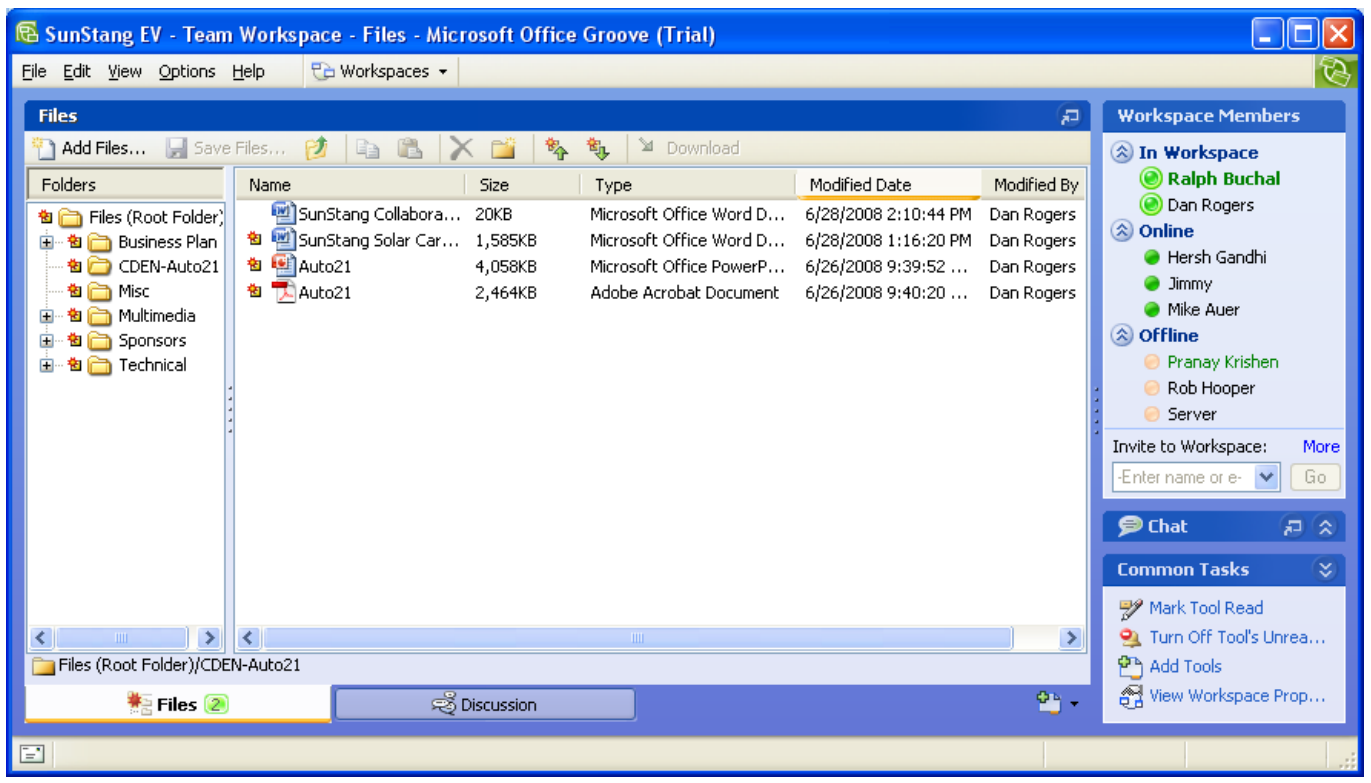

Figure 3. Example of Groove Workspace. 
application rather than a Web interface. This provides additional Windows functionality, including full support for drag and drop of files. A notification system informs all users when a change has been made to the shared files (new files added, existing files modified, etc.). Furthermore, it is possible to create individual 'workspaces' for sub-teams. Groove includes additional collaboration tools such as discussion forums, chat, shared calendars, shared sketchpad, task manager, photo gallery, and meeting manager.

Groove's peer-to-peer architecture has advantages and disadvantages. On the plus side, this type of network eliminates the need to maintain a file server, and synchronizes all files on the local hard-drives of each user, making them available even off-line. However, the disadvantage is that there are multiple copies of each file - one on each client computer. There is no version control or check-in/check-out functionality, which makes it difficult for larger groups working on the same document. When multiple users edit the same document while off-line, versions will be overwritten in an unpredictable way as users go on-line and sync their files. Also, if a file is deleted by a user, it is deleted from all user computers with no possibility of recovery.

An additional challenge is that Groove must be installed on each client machine. Groove can be purchased separately, or bundled as part of Microsoft Office 2007 Enterprise Edition. The educational cost of this bundle is no more than many textbooks, so this is not a major hurdle.

An example Groove workspace is shown in Figure 4.

\subsection{Microsoft OneNote}

Microsoft OneNote [5] is an application for duplicating and extending the capabilities of a paper notebook. Like a real notebook, OneNote allows notes to be organized by Notebooks, Section Tabs, and pages. With a TabletPC, users can use it just like a real notebook, using freehand text and diagrams. In addition to freehand entry, users can use all of the usual computer tools to enter and format text, create diagrams, insert graphics and images, etc. All of the notebook content can be rearranged on the page as

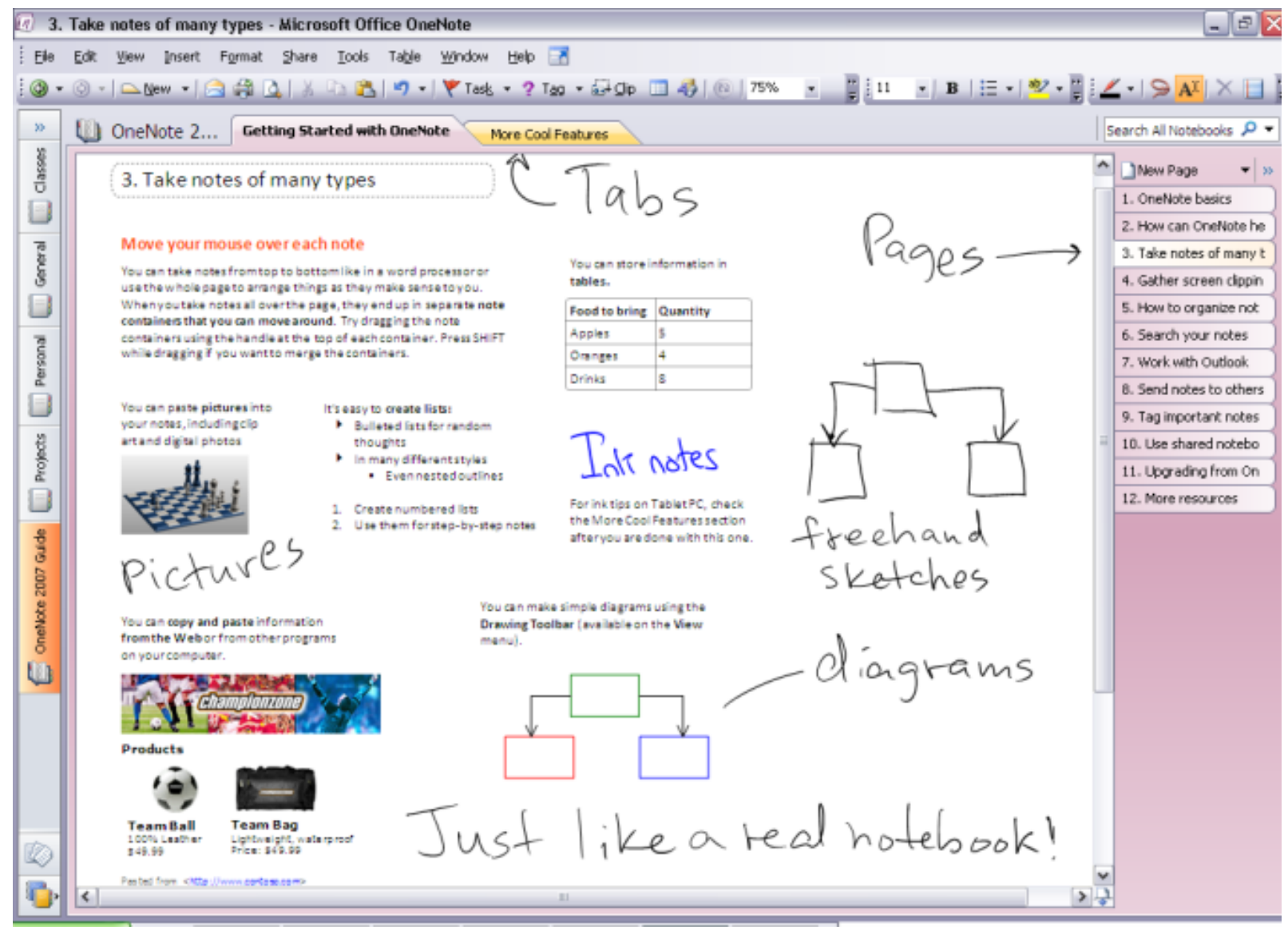

Figure 5. Sample OneNote page. 
desired. A sample notebook page is shown in Figure 5.

OneNote can support simultaneous and asynchronous shared document editing. For sharing, OneNote requires that the Notebook files be stored on a shared network location accessible by all team members. OneNote synchronizes modifications made by all team members, and maintains a single document that merges all of the changes. However, OneNote does not track versions, and does not record change history by user.

OneNote tracks changes by date, and allows recent changes to be identified. It also supports tagging, with content searchable by tag type. Examples of tag types include "ideas", and "questions".

OneNote does not provide automatic user identification during simultaneous editing sessions, so users must indicate their changes via real-time chat or other ad hoc methods. OneNote also has no provision for checkin/check-out to manage changes to the notebook.

Like Groove, OneNote is bundled as part of Microsoft Office 2007 Enterprise Edition.

\subsection{PDMWorks}

PDMWorks Workgroup [6] is a basic PDM tool from SolidWorks Corp. It provides the necessary functionality to manage SolidWorks files, and it is easy to administer and use [7]. PDMWorks includes many standard document management capabilities including version control and check-in/check-out. It can manage files of any type, but is intended for managing SolidWorks CAD files. SunStang and other teams have found PDMWorks to be an effective way to manage CAD files. The main disadvantage of PDMWorks Workgroup is its poor scalability to larger teams.

\section{Conclusions and Recommendations}

Based on the requirements of the upcoming SunStang design cycle, team management has opted to use a combination of Google Apps and Microsoft Groove. Google Apps integrates well with the team's existing @sunstang.ca email accounts, and provides a centralized user account management interface for related collaboration tools. Google Apps is available at no cost and it requires no IT support. The team's file sharing needs will be addressed through the use of Microsoft Groove rather than relying on a server requiring IT support. PDMWorks Workgroup will be used to manage SunStang CAD data.

An important factor in these decisions was the desire to avoid relying on Faculty or University IT infrastructure and support. Existing institutional IT infrastructure generally focuses on basic services like email and websites, and often the need for more sophisticated collaboration infrastructure is not recognized as a priority. Evaluation and implementation of collaboration tools should not be left to student teams, but should be part of institutional strategic planning.

\section{References}

[1] Yahoo! Canada Groups, Yahoo! Canada Co., http://ca.groups.yahoo.com/, Accessed June 30, 2008.

[2] Microsoft Office SharePoint Server, Microsoft, http://www.microsoft.com/Sharepoint/default.mspx, accessed June 30, 2008.

[3] Google Apps, Google Inc., http://www.google.com/a/help/intl/en/business/applications.h

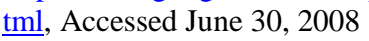

[4] Microsoft Office Groove, Microsoft Office Online, http://office.microsoft.com/en-us/groove/default.aspx, accessed June 30, 2008.

[5] Microsoft Office OneNote, Microsoft Office Online, http://office.microsoft.com/en-us/onenote/default.aspx, Accessed June 30, 2008.

[6] PDMWorks Workgroup, SolidWorks Corp., http://www.solidworks.com/Pages/products/solutions/pdmwo rks.html, Accessed June 30, 2008.

[7] Buchal, R. O., The Use of Product Data Management (PDM) Software to Support Student Design Projects, The $3^{r d}$ CDEN/RCCI International Design Conference, Toronto, Ontario, July 24-26, 2006 